\title{
Trying to Explain the Theoretical Basis of Traditional Chinese Medicine "Huashi Baidu Fang" in the Treatment of Coronavirus Disease 2019 with Western Medical Theory: A Review
}

\author{
Dandan Song, Hualiang Chen* \\ Department of Pharmacy, Shaoxing Seventh People's Hospital, Shaoxing, China \\ Email: *chenxianzhi@tom.com; *chenxianzhi2000@aliyun.com
}

How to cite this paper: Song, D.D. and Chen, H.L. (2020) Trying to Explain the Theoretical Basis of Traditional Chinese Medicine "Huashi Baidu Fang" in the Treatment of Coronavirus Disease 2019 with Western Medical Theory: A Review. $A d$ vances in Bioscience and Biotechnology, 11, 421-441.

https://doi.org/10.4236/abb.2020.119029

Received: August 6, 2020

Accepted: September 14, 2020

Published: September 17, 2020

Copyright $\odot 2020$ by author(s) and Scientific Research Publishing Inc. This work is licensed under the Creative Commons Attribution International License (CC BY 4.0).

http://creativecommons.org/licenses/by/4.0/

\begin{abstract}
Purpose: This work is aimed to explain the theoretical basis of "Huashi Baidu Fang" in the treatment of coronavirus disease 2019 (COVID-19) with western medical theory. Methods: We analyze the "Diagnosis and Treatment Protocol for COVID-19 (Version 1 to Version 7)" made by China, "Clinical management of severe acute respiratory infection when novel coronavirus (2019-nCoV) infection is suspected-Interim guidance" made by World Health Organization (WHO), "Therapeutic Guidelines: Respiratory (Version 5)", "Therapeutic Guidelines: Gastrointestinal (Version 5)" and "Therapeutic Guidelines: Antibiotic (Version 15)" published by Australia, and the origin of classical prescription of "Huashi Baidu Fang": "Shanghanlun (Treatise on Febrile Diseases)", "Jinkui Yaolue (Synopsis of Golden Chamber)" and "Wenyi Lun (The Epidemic Febrile Disease)". We search the dictionary of traditional Chinese medicine (Version II) manually. And we search literatures from 2001 to 2020 on Wiley online library. We conduct a comparative study among the symptoms of TCM formulations in "Huashi Baidu Fang", the pathogenesis and clinical manifestation of COVID-19 and COPD with acute gastrointestinal inflammation. And we carry out pharmacological inquiry of "Huashi Baidu Fang". Results: The clinical manifestations of respiratory symptoms and gastrointestinal tract of syndrome of lung obstruction due to epidemic toxin and acute exacerbation of COPD are almost the same; The formulations used in "Huashi Baidu Fang" are consistent with the pharmacological activity of the drug recommended in the Therapeutic Guidelines. Conclusion: "Huashi Baidu Fang" may play a positive role in COVID-19.
\end{abstract}

\section{Keywords}

Huashi Baidu Fang, COVID-19, COPD, Gastrointestinal Inflammation 


\section{Introduction}

Since COVID-19 has a breakout in Wuhan, China, in December 2019, more than 9 million people have been diagnosed with COVID-19 globally and more than 400,000 have died. The epidemic of COVID-19 has seriously affected the political, economic and social operation of different countries around the world, seriously affected people's work, life and study, and more seriously affected people's life, health and safety.

We take "Huashi Baidu Fang" as a research subject to study the origin of classical prescription of "Huashi Baidu Fang": "Shanghan Lun (Treatise on Febrile Diseases)" and "Jinkui Yaolue (Synopsis of Golden Chamber)" of Zhang Zhongjing in Han Dynasty, and "Wenyi Lun (The Epidemic Febrile Disease)" by Wu Youxing in Ming Dynasty, and to study the theoretical basis of traditional Chinese medicine such as pathogenesis, clinical manifestations, symptoms, diagnosis and treatment methods. We take "Diagnosis and Treatment Protocol for COVID-19 (Version 1 to Version 7)" made by China, "Clinical management of severe acute respiratory infection when novel coronavirus (2019-nCoV) infection is suspected-Interim guidance" made by World Health Organization (WHO), "Therapeutic Guidelines: Respiratory (Version 5)", "Therapeutic Guidelines: Gastrointestinal (Version 5)" and "Therapeutic Guidelines: Antibiotic (Version 15)" published by Australia as control subjects to compare and analyze "Huashi Baidu Fang", the pathogenesis, clinical manifestations, diagnosis and treatment of acute exacerbations of COPD with functional gastrointestinal diseases and acute gastroenteritis caused by COVID-19. The pharmacological effects of the prescription of TCM are verified, and the corresponding pathologies and diseases caused by COVID-19 are studied. We try to explain the theoretical basis of "Huashi Baidu Fang" in the treatment of coronavirus disease 2019 (COVID-19) with western medical theory and its effectiveness and relative scientificity.

\section{Materials and Methods}

\subsection{Materials}

Research refers to analysis the "Diagnosis and Treatment Protocol for Novel Coronavirus Pneumonia" (Version 1 to Version 7) made by China, "Clinical management of severe acute respiratory infection when novel coronavirus (2019-nCoV) infection is suspected-Interim guidance" made by World Health Organization (WHO), “Therapeutic Guidelines: Respiratory (Version 5)"; "Therapeutic Guidelines: Gastrointestinal (Version 5)" and "Therapeutic Guidelines: Antibiotic (Version 15)": respiratory infection: acute exacerbation of chronic obstructive pulmonary disease (COPD); gastrointestinal infection: acute gastroenteritis published by Australia.

This study analyzes the classical prescription of "Huashi Baidu Fang": "Shanghan Lun (Treatise on Febrile Diseases)", "Jinkui Yaolue (Synopsis of Golden Chamber)", and "Wenyi Lun (The Epidemic Febrile Disease)". "Shanghan Lun 
(Treatise on Febrile Diseases)" is a reference to the photocopied edition of Baiyunge-“Shanghan Zabing Lun", "Shanghan Lun Jiaozhu" published by People's Medical Publishing House edited by Liu Duzhou, and "Shanghan Lun Yandu" which is the textbook for National high education in the field of traditional Chinese medicine. "Jinkui Yaolue (Synopsis of Golden Chamber)" is comparative with "Jinkui Yaolue Jiaozhu" published by People's Medical Publishing House edited by Li Keguang. "Wenyi Lun (The Epidemic Febrile Disease)" is compared with "Wenyi Lun (version 2)" published by the Chinese medical science and technology press of Wu Youke's notes on He Yong, and "Wenyi Lun Pingzhu" published by people's medical publishing house, commented by the institute of traditional Chinese medicine of Zhejiang Province.

The formulation theory and drug composition are studied according to the clinical manifestations of the prescription which is divided into respiratory system and gastrointestinal system by referring to different academic thoughts of "Shanghan Lun (Treatise on Febrile Diseases)"; "Jinkui Yaolue (Synopsis of Golden Chamber)" of Zhang Zhongjing, and "Wenyi Lun (The Epidemic Febrile Disease)" by Wu Youxing. According to the source of literature, "Shanghan Lun (Treatise on Febrile Diseases)" is divided into Mahuang Xingren Gancao Shigao decoction; "Jinkui Yaolue (Synopsis of Golden Chamber)" is divided into Tingli Dazao Xiefei decoction and Houpo Dahuang decoction; "Wenyi Lun (The Epidemic Febrile Disease)" is divided into Dayuan Yin and Dahuang decoction, Banxia Huoxiang decoction.

We search the basic decoction pieces in the dictionary of traditional Chinese medicine (Version II) manually, which is published by Shanghai science and technology publishing house and redacted by Nanjing University of Traditional Chinese Medicine. The botanical origin, chemical composition and pharmacology of basic decoction pieces are verified. The pharmacology of prescription drugs in dictionary is confirmed and the dictionary is cited until 2001.

We use the Internet to search related literatures from 2001 to 2020 on Wiley online library (https://onlinelibrary.wiley.com). The botanical origin, chemical composition and pharmacology of basic decoction pieces are verified.

\subsection{Methods}

The retrieval results are summarized and recorded. And a comparative study is conducted on the pathogenesis, clinical manifestations, symptomatic diagnosis, therapeutic methods and pharmacology of TCM and western medicine.

\section{Results}

\subsection{The Contrast among TCM Formula Symptoms of "Huashi Baidu Fang", Acute Exacerbation of COPD and the Pathogenesis and Clinical Manifestation of COVID-19}

"Huashi Baidu Fang" is a TCM prescription for syndrome of lung obstruction due to epidemic toxin. The clinical manifestation of syndrome of lung obstruc- 
tion due to epidemic toxin divides into respiratory tract and gastrointestinal tract. Clinical manifestations of respiratory tract are as follows: fever, cough, expectoration, polypnea, gasp, purulent sputum, blood-stained sputum, breath with difficulty and lacking in strength. The clinical manifestations of acute exacerbation of COPD are as follows: shortness of breath is aggravating, polypnea, reduction of activity tolerance, cough frequently, purulent sputum, with or without fever, right heart failure, ankle edema. The clinical manifestations of respiratory symptoms of syndrome of lung obstruction due to epidemic toxin and acute exacerbation of COPD are almost the same (Table 1).

The clinical manifestations of gastrointestinal tract are as follows: fever, nausea, dyspepsia, inhibited defecation, frequent micturition. Gastrointestinal infection in Therapeutic Guidelines: symptoms of acute gastroenteritis last less than 14 days, a high fever caused mainly by a viral or bacterial infection, leukocytosis, abdominal tenderness or severe abdominal pain, diarrhea with hypovolemia, bloody stools and emesis, etc. Symptoms of functional gastrointestinal disease in Therapeutic Guidelines include dysfunction of upper gastrointestinal tract, nausea and vomiting and dysfunction of lower digestive tract; dysfunction of upper gastrointestinal tract, nausea and vomiting with functional heartburn, functional dyspepsia, belching, hiccup, nausea and vomiting. Symptoms of lower digestive tract disorders are as follows: irritable bowel syndrome, functional constipation, functional diarrhea, and functional bloating. Clinical manifestations of gastrointestinal tract of syndrome of lung obstruction due to epidemic toxin correspond to functional gastrointestinal disease, infection of gastrointestinal and acute gastroenteritis in Therapeutic Guidelines (Table 2).

\subsection{Comparison of TCM Formula Symptoms of "Huashi Baidu Fang" and Therapeutic Methods of Acute Exacerbation of COPD with Acute Gastrointestinal Inflammation}

\subsubsection{Comparison of TCM Formula Symptoms of "Huashi Baidu Fang" and Diagnosis and Therapeutic Methods of Acute Exacerbation of COPD}

Acute exacerbation of COPD in Therapeutic Guidelines are treated by inhaling bronchodilators to relieve symptoms, long-acting bronchodilators reduce acute exacerbations, using systemic glucocorticoids to shorten hospital stays, promote recovery of lung function to baseline, and control symptoms well, using antibiotics to treat secondary infections. If the patient has hypoxemia, oxygen therapy to bring the blood oxygen saturation to $88 \%-93 \%$, and mechanical ventilation is used if the patients are with type II respiratory failure and acidosis.

TCM formula symptoms of "Huashi Baidu Fang": Zhang Zhongjing discussed exogenous typhoid fever in "Shanghan Lun (Treatise on Febrile Diseases)": symptoms include headache, stiff neck, fear of cold, fever or no fever, and asthma after sweating, using Mahuang Xingren Gancao Shigao Decoction to treat these symptoms. In "Jinkui Yaolue (Synopsis of Golden Chamber)", Zhang Zhongjing argued that if the disease progresses to pulmonary inflammation and secondary 
Table 1. Comparison of the clinical manifestations of respiratory system with the TCM classics, guidelines, Chinese protocol, temporary WHO documents and pathological changes.

\begin{tabular}{|c|c|c|c|c|c|}
\hline $\begin{array}{l}\text { The clinical } \\
\text { manifestations of } \\
\text { syndrome of lung } \\
\text { obstruction due to } \\
\text { epidemic toxin } \\
\text { described in Chinese } \\
\text { protocol for severe }\end{array}$ & $\begin{array}{l}\text { The symptoms } \\
\text { described in traditional } \\
\text { Chinese medicine }\end{array}$ & $\begin{array}{l}\text { Therapeutic } \\
\text { Guidelines: } \\
\text { Symptoms of COPD }\end{array}$ & $\begin{array}{l}\text { Chinese } \\
\text { protocol clinical } \\
\text { classification of } \\
\text { symptoms }\end{array}$ & $\begin{array}{l}\text { Pathology } \\
\text { changes in } \\
\text { Chinese } \\
\text { protocol }\end{array}$ & $\begin{array}{l}\text { WHO Temporary Document: } \\
\text { Acute respiratory distress } \\
\text { syndrome symptoms (ARDS) }\end{array}$ \\
\hline
\end{tabular}

"Jinkui Yaolue (Synopsis of Golden Chamber)" Cough, Clinical manifestations: chest pain, cough sputum, hot and red face, cough, sputum has pus and blood, yellow and sticky asthma, cannot lie flat, must phlegm breathing on the bed, ascites, abdominal distension, and constipation
Moderate cases:

increased shortness of breath, short of breath during walking, limited daily life, cough and expectoration, acute exacerbation requires glucocorticoids. FEV1 $\approx 40 \%-59 \%$ estimated value
"Wenyi Lun (The Epidemic

Febrile Disease)": At the

beginning of the epidemic, Severe cases: people were afraid of cold shortness of breath before they got fever. Later, after slight activity, they only felt hot but were severe restriction of not afraid of cold. Early daily activities,

Or sputum with illness 2 - 3 days, day and frequent blood, breathless and night fever, headache body expectoration of suffocated, and tired pain, white moss on the sputum, chronic tongue. In the middle, the cough, coating on the tongue will glucocorticoid for turn yellow and infect the acute exacerbation, stomach. Heavy stage of FEV1 $<40 \%$ yellow tongue, Heart stuffy, estimated value abdominal distension, and vomiting
Common cases: with fever, respiratory symptoms, imaging findings of pneumonia and transparent membrane were found in alveolar cavity. Alveolar septal vascular congestion, edema, mononuclear and lymphocyte infiltration and

Severe cases: Adults who conform to any of the following criteria: 1) Onset of shortness of breath, RRN30 times/min; 2 In resting state, oxygen saturation < 93\%; 3) Arterial partial pressure of oxygen $\left(\mathrm{PaO}_{2}\right)$ /fraction of inspired oxygen $\left(\mathrm{FiO}_{2}\right)<300 \mathrm{mmHg}$ $(1 \mathrm{mmHg}=0.133$ $\mathrm{kPa}$ ) Cases with chest imaging that shows obvious lesion progression within 24 - 48 hours $>50 \%$ shall be managed as severe clear thrombosis can be seen. Lung tissue focal hemorrhage, necrosis, can appear

hemorrhagic infarction. Partial alveolar exudation and pulmonary interstitial fibrosis. Part of the bronchial mucosa epithelium in the lung was detached, and mucous and mucus plug were found in the cavity. A few alveolar hyperinflation, alveolar septal rupture or cysts formed
Onset: New respiratory symptoms or aggravation of existing symptoms appear within one week after the diagnosis of clinical disease. Chest imaging: Bilateral pulmonary turbidity, which cannot be fully explained by pulmonary effusion, lobar or pulmonary atrophy, or pulmonary nodules. Cause of edema: respiratory failure, which cannot be fully explained by heart failure or fluid overload. If there are no risk factors, an objective assessment (e.g. echocardiography) should be performed to exclude hydrostatic pressure edema. Degree of oxygenation (adult):

- Mild ARDS: $200 \mathrm{mmHg}<$ $\mathrm{PaO}_{2} / \mathrm{FiO}_{2} \leq 300 \mathrm{mmHg}(\mathrm{PEEP}$ or $\mathrm{CPAP} \geq 5 \mathrm{~cm} \mathrm{H}_{2} \mathrm{O}$ or nonmechanical ventilation) Moderate ARDS: $100 \mathrm{mmHg}<$ $\mathrm{PaO}_{2} / \mathrm{FiO}_{2} \leq 200 \mathrm{mmHg}(\mathrm{PEEP}$ $\geq 5 \mathrm{cmH}_{2} \mathrm{O}$ or nonmechanical ventilation)

- Severe ARDS: $\mathrm{PaO}_{2} / \mathrm{FiO}_{2} \leq 100$ $\mathrm{mmHg}\left(\mathrm{PEEP} \geq 5 \mathrm{cmH}_{2} \mathrm{O}\right.$ or nonmechanical ventilation) When $\mathrm{PaO}_{2}$ value is not available, $\mathrm{SpO}_{2} / \mathrm{FiO}_{2} \leq 315$ indicates ARDS (including patients without mechanical ventilation)

- Degree of oxygenation (Children; Note: OI = Oxygenation Index, OSI $=$ Oxygenation index using $\mathrm{SpO}_{2}$ )

- Positive pressure noninvasive ventilation or full face mask CPAP $\geq 5 \mathrm{~cm} \mathrm{H} \mathrm{O}_{2}: \mathrm{PaO}_{2} / \mathrm{FiO}_{2} \leq$ $300 \mathrm{mmHg}$ or $\mathrm{SpO}_{2} / \mathrm{FiO}_{2} \leq 264$

- Mild ARDS (Invasive ventilation): $4 \leq$ OI $<8$ or $5 \leq$ OSI $<7.5$

- Moderate ARDS (Invasive ventilation): $8 \leq$ OI $<16$ or $7.5 \leq$ OSI $<12.3$

- Severe ARDS (Invasive ventilation): $\mathrm{OI} \geq 16$ or $\mathrm{OSI} \geq$ 12.3 
Table 2. Comparison of clinical manifestations and pathological changes in gastrointestinal system of Huashi Baidu Fang with TCM classics, guidelines, Chinese protocol, temporary WHO documents.

\begin{tabular}{|c|c|c|c|c|}
\hline $\begin{array}{l}\text { The clinical manifestations } \\
\text { of syndrome of lung } \\
\text { obstruction due to epidemic } \\
\text { toxin described in } \\
\text { Chinese protocol }\end{array}$ & $\begin{array}{l}\text { The symptoms described in } \\
\text { traditional Chinese medicine }\end{array}$ & $\begin{array}{l}\text { Therapeutic Guidelines: Acute } \\
\text { gastroenteritis } \\
\text { symptoms of gastrointestinal } \\
\text { tract infection in functional } \\
\text { gastrointestinal diseases }\end{array}$ & $\begin{array}{l}\text { WHO Provisional } \\
\text { Document: Acute } \\
\text { respiratory distress } \\
\text { syndrome (ARDS) }\end{array}$ & $\begin{array}{l}\text { Changes of pathology in } \\
\text { Chinese protocol }\end{array}$ \\
\hline $\begin{array}{l}\text { Clinical manifestations: } \\
\text { dryness, bitterness and } \\
\text { stickiness in the mouth, } \\
\text { nausea and loss of appetite, } \\
\text { difficult defecation, and scanty } \\
\text { dark urine. The tongue is red } \\
\text { with yellow greasy coating. } \\
\text { The pulse is slippery and } \\
\text { rapid }\end{array}$ & $\begin{array}{l}\text { "Wenyi Lun (The Epidemic } \\
\text { Febrile Disease)": at the } \\
\text { beginning of the epidemic, } \\
\text { people were afraid of cold } \\
\text { before they got fever. Later, } \\
\text { they only felt hot but were not } \\
\text { afraid of cold. During the } 2 \text { - } 3 \\
\text { days of the initial illness, } \\
\text { patients have fever day and } \\
\text { night, headache and body pain, } \\
\text { and white lichen on the tongue. } \\
\text { Yellow, yellow coating on the } \\
\text { tongue, heart stuffy, flatulence } \\
\text { and vomiting }\end{array}$ & $\begin{array}{l}\text { Functional gastrointestinal } \\
\text { diseases include irritable bowel } \\
\text { syndrome, functional } \\
\text { constipation, and upper } \\
\text { gastrointestinal disorders. } \\
\text { Symptoms include functional } \\
\text { dyspepsia, nausea, vomiting, } \\
\text { abdominal distension, upper } \\
\text { fullness, early satiety, } \\
\text { abdominal pain, functional } \\
\text { diarrhea, functional } \\
\text { constipation, and functional } \\
\text { abdominal distension } \\
\text { Gastrointestinal infection: The } \\
\text { clinical manifestations of acute } \\
\text { gastroenteritis are the } \\
\text { symptoms lasting less than } 14 \\
\text { days, mainly due to viral or } \\
\text { bacterial infection caused by } \\
\text { the increase of hot white blood } \\
\text { cells, abdominal tenderness or } \\
\text { severe abdominal pain, massive } \\
\text { diarrhea with low blood } \\
\text { volume, blood stool and } \\
\text { vomiting }\end{array}$ & $\begin{array}{l}\text { Patients with } \\
\text { anorexia, very few } \\
\text { patients may present } \\
\text { with diarrhea, } \\
\text { nausea and vomiting } \\
\text { gastrointestinal } \\
\text { symptoms }\end{array}$ & $\begin{array}{l}\text { The mucosa epithelium of } \\
\text { esophagus, stomach and } \\
\text { intestine degenerate, } \\
\text { necrose and exfoliate in } \\
\text { different degrees. } \\
\text { Histologically, there are } \\
\text { occasional lymphocytic } \\
\text { infiltrates in the squamous } \\
\text { epithelium of the } \\
\text { esophagus, and numerous } \\
\text { infiltrating plasma cells } \\
\text { and lymphocytes with } \\
\text { stromal edema in the } \\
\text { lamina propria of the } \\
\text { stomach, duodenum, and } \\
\text { rectum [37] }\end{array}$ \\
\hline
\end{tabular}

infection, symptoms include cough chest pain, cough sputum, sputum has pus and blood, asthma cannot stay in bed, use Tingli Dazao Xiefei decoction to treat them. If the disease develops to cough and asthma, cannot lie flat, must breathe on the bed, there is ascites between the chest diaphragm, abdominal distension, constipation, use Houpo Dahuang Decoction for treatment. The process of causing dyspnea, aggravation of inflammation, production of ascites and infection to the gastrointestinal tract is basically consistent with the Therapeutic Guidelines. The formulations used (in the functional group form of the drug) are consistent with the pharmacological activity of the drug recommended in the Therapeutic Guidelines (3.4).

\subsubsection{Comparison of TCM Formula Symptoms of "Huashi Baidu Fang" and the Diagnosis and Treatment of Gastrointestinal Diseases}

Therapeutic guidelines for acute gastroenteritis: viral gastroenteritis supplementing fluids and electrolytes, bacterial gastroenteritis supplementing fluids 
and electrolytes, and toxin-mediated gastroenteritis using supportive therapy. Antibiotic therapy is unnecessary and inappropriate for most patients with bacterial diarrhea. Empirical antibiotic treatment is used when necessary. The onset time of gastrointestinal infection caused by COVID-19 is short, the pathogeny is infection or secondary infection, and there is an inflammatory reference, so we refer to gastrointestinal infection.

Functional gastrointestinal diseases in Therapeutic Guidelines include upper gastrointestinal disorders and lower gastrointestinal disorders. The treatment of upper gastrointestinal dysfunction, nausea and vomiting is as follows: moderate to severe nausea and vomiting are treated with vitB6 and one of the next drugs: doxilamine, metoclopramide and promethazine, when the effect is not satisfactory using ondansetron. Functional dyspepsia is treated with proton pump inhibitors and domperidone. Gastroparesis is treated with domperidone, metoclopramide and cisapride. Loperamide and coretenine are used for the treatment of lower gastrointestinal disorders. Scopolamine butyrobromide, mebelverine, peppermint oil and antidepressants are used for the treatment of abdominal pain, and laxatives are used for the treatment of constipation.

In "Jinkui Yaolue (Synopsis of Golden Chamber)", Zhang Zhongjing argued that If the disease develops to cough and asthma, unable to lie flat, must breathe on the bed, ascites, abdominal distension, constipation, use Houpo Dahuang Decoction for treatment (3.2.1). In "Wenyi Lun (The Epidemic Febrile Disease)", Wu Youxing discussed that in the early stage of the epidemic, he used Dayuan Yin in the morning and evening to promote the collapse of the evil spirit, inhibit the development of infection, and expel the evil spirit from the body to achieve the purpose of treatment. If the tongue coating becomes yellow and have a infection to the stomach, must add Houpo Dahuang Decoction. If the symptoms appear yellow tongue and stuffy heart and full abdomen, increase the amount of Dahuang (Radix et Rhizoma Rhei) on the basis of Dayuan Yin to promote gastrointestinal drainage, remove the qi and blood stasis caused by evil qi in the gastrointestinal tract, and eliminate it from the intestine. If vomiting after the elimination of intestinal evil with Dahuang (Radix et Rhizoma Rhei), use Banxia Huoxiang Decoction for antiemetic. The formulations used (in the functional group form of the drug) are consistent with the pharmacological activity of the drug recommended in the Therapeutic Guidelines (3.4).

\subsection{Etiology}

In "Wenyi Lun (The Epidemic Febrile Disease)", Wu Youxing discussed that the disease has been infected with pestilential qi, which has mouth and nose transmission and contact transmission, whether old, young, strong or weak, will be infected. If the healthy gas slightly decline, infection would come on. The pestilential qi enters from the mouth and nose and attaches to the membrane not far from the stomach. Wu Youxing stressed that if the coating on the tongue has turned yellow, it would infect the stomach. Wu's discussion is basically similar to the transmission mode of COVID-19. 


\subsection{The Pharmacology Basis of TCM Formula in "Huashi Baidu Fang" (Table 3(a) and Table 3(b))}

The basic prescription of "Huashi Baidu Fang": Shengmahuang (Herba Ephedrae), Xingren (Semen Armeniacae Amarum), Shengshigao (Gypsum Fibrosum), Gan Cao (Radix Glycyrrhizae), Huoxiang (Herba Pogostemonis), Houpo (Cortex Magnoliae Officinalis), Cangzhu (Rhizoma Atractylodis), Caoguo (Fructus Tsaoko), Fabanxia (Rhizoma Pinelliae preparatum), Fuling (Poria cocos), Shengdahuang (Radix et Rhizoma Rhei), Shenghuangqi (Radix Astragali), Tinglizi (Lepidium apetalum Willd), Chishao (Radix Paeoniae Rubra).

Table 3. (a) Pharmacology basis of Dayuan Yin and Xiaochengqi decoction; (b) Pharmacology basis of Huashi Baidu Fang.

(a)

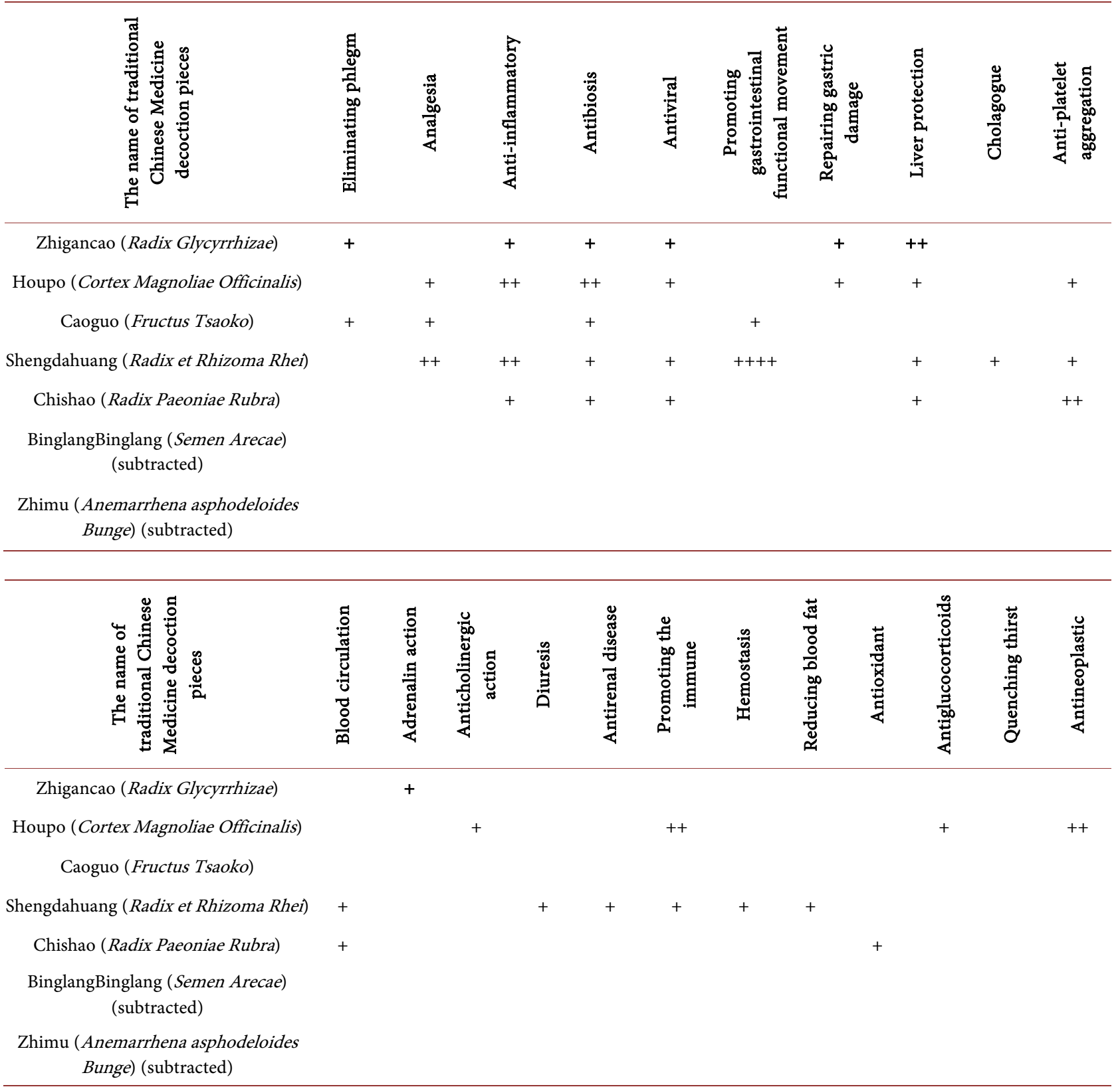


(b)

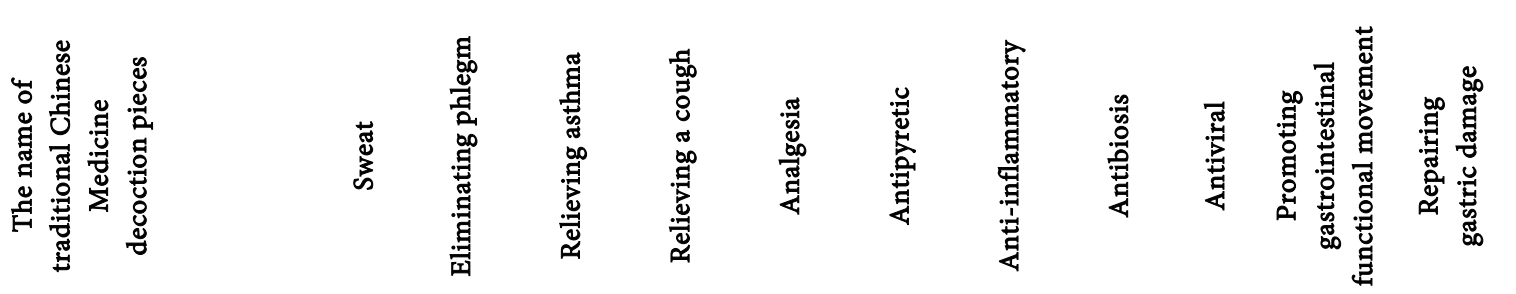

\begin{tabular}{|c|c|c|c|c|c|c|c|c|c|c|}
\hline Mahuang (Herba Ephedrae) & + & + & & & + & + & & & + & \\
\hline Zhigancao (Radix Glycyrrhizae) & + & & & & & + & + & + & & + \\
\hline Xingren (Semen Armeniacae Amarum) & & + & & + & & + & & & + & \\
\hline Shengshigao (Gypsum Fibrosum) & & & & & + & & & & & \\
\hline Huoxiang (Herba Pogostemonis) & & & & + & & & ++ & + & + & \\
\hline Houpo (Cortex Magnoliae Officinalis) & & & & + & & ++ & ++ & + & & + \\
\hline Cangzhu (Rhizoma Atractylodis) & & & & & & & ++ & + & ++ & ++ \\
\hline Caoguo (Fructus Tsaoko) & + & & & + & & & + & & + & \\
\hline Fabanxia (Rhizoma Pinelliae preparatum) & & + & + & & & + & & & & + \\
\hline Fuling (Poria cocos) & & & & & & + & & & & + \\
\hline Shengdahuang (Radix et Rhizoma Rhei) & & & & ++ & & ++ & + & + & ++++ & \\
\hline Shenghuangqi (Radix Astragali) & & & & & & ++ & & + & & \\
\hline Tinglizi (Lepidium apetalum Willd) & & + & + & & & & + & & & \\
\hline Chishao (Radix Paeoniae Rubra) & & & & & & + & + & + & & \\
\hline
\end{tabular}

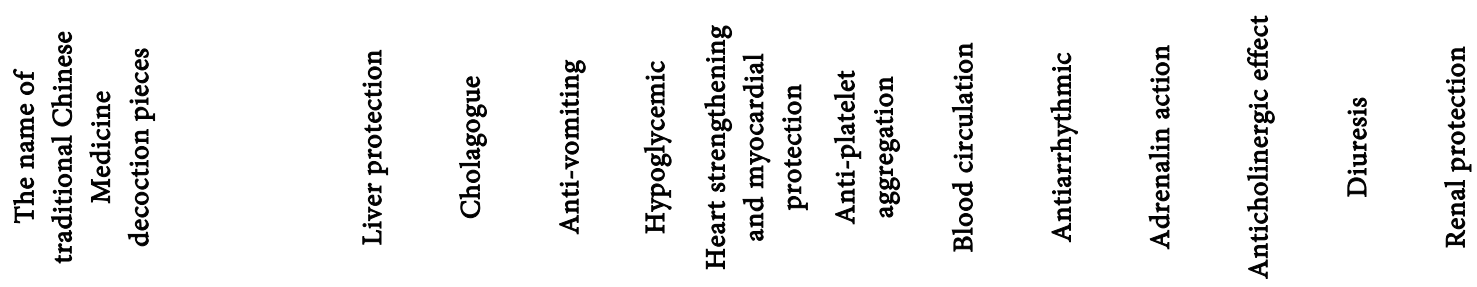

Mahuang (Herba Ephedrae)

Zhigancao (Radix Glycyrrhizae)

Xingren (Semen Armeniacae Amarum)

Shengshigao (Gypsum Fibrosum)

Huoxiang (Herba Pogostemonis)

Houpo (Cortex Magnoliae Officinalis)

Cangzhu (Rhizoma Atractylodis)

Caoguo (Fructus Tsaoko)

Fabanxia (Rhizoma Pinelliae preparatum)

Fuling (Poria cocos)

Shengdahuang (Radix et Rhizoma Rhei)

Shenghuangqi (Radix Astragali)

Tinglizi (Lepidium apetalum Willd)

Chishao (Radix Paeoniae Rubra) 


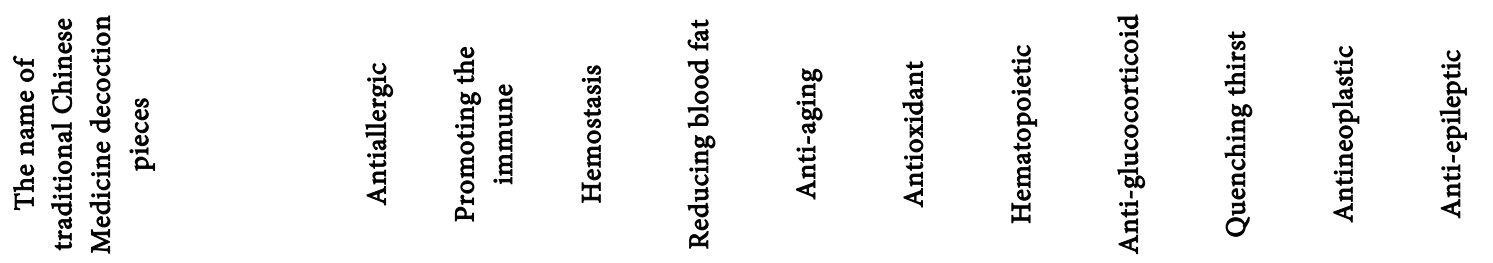

Mahuang (Herba Ephedrae)

Zhigancao (Radix Glycyrrhizae)

Xingren (Semen Armeniacae Amarum)

Shengshigao (Gypsum Fibrosum)

Huoxiang (Herba Pogostemonis)

Houpo (Cortex Magnoliae Officinalis)

Cangzhu (Rhizoma Atractylodis)

Caoguo (Fructus Tsaoko)

Fabanxia (Rhizoma Pinelliae preparatum) Fuling (Poria cocos)

Shengdahuang (Radix et Rhizoma Rhei)

Shenghuangqi (Radix Astragali)

Tinglizi (Lepidium apetalum Willd)

Chishao (Radix Paeoniae Rubra)

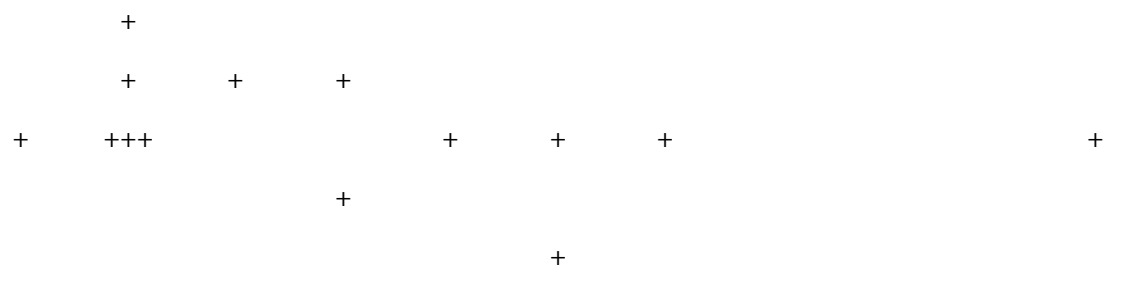

1) Mahuang (Herba Ephedrae) Ephedrine is the main component of Mahuang. Its main pharmacological action is stimulating the central nervous system, relieving bronchial smooth muscle spasm, relieving cough and asthma, and dispelling phlegm [1] [2].

2) Xingren (Semen Armeniacae Amarum) The main pharmacological action is inhibiting the cough center and playing antitussive effect [3] [4].

3) Gan Cao (Radix Glycyrrhizae) The main pharmacological action is adrenocortical hormone like action, fighting ventricular arrhythmia and reduce of ventricular fibrillation action [5]. It has analgesic, antipyretic and sedative effects on the central nervous system. It also has anti-peptic ulcer, anti-intestinal spasm, expectorant and antitussive effect. Glycyrrhizin enhances the non-specific immune function, has the synergistic effect with the glucocorticoid, and has the effect of liver protection and detoxification [5]. Besides, it has antiviral activity and anti-methicillin-resistant staphylococcus aureus activity [6].

4) Huoxiang (Herba Pogostemonis) The main ingredient is pogostemon alcohol, which can promote the secretion of gastric juice, enhance the digestive power, and have the effect of relieving the spasm in stomach and intestine [7]. It has antifungal and antibacterial activities, anti-atherosclerosis effect, expansion of microvessels and slight sweating effect [8].

5) Houpo (Cortex Magnoliae Officinalis) Magnolol shows significant liver protection and tumor inhibition [9]. Honokiol has a strong inhibition of a va- 
riety of inflammatory responses [10]. It has strong anti-hepatitis $\mathrm{C}$ virus activity at non-toxic concentration [11], and has protective effect on the intestinal injury induced by oxaliplatin in mice [12]. Magnolol and Honokiol has antibacterial activities against vancomycin-resistant and methicillin-resistant Staphylococcus aureus at the lowest concentration, anti-biofilm and bactericidal effects [13], significant central inhibitory effect, significant inhibition of platelet aggregation and ATP release and neuroprotective effect [14]. Magnolol also has anti-depressants, anti-anxiety and sleep aids effects [15].

6) Cangzhu (Rhizoma Atractylodis) The volatile oil of Cangzhu (Rhizoma Atractylodis) has the function of anti-intestinal spasm, relaxation of enteric muscle, promoting gastrointestinal movement, and weak contraction of gastric smooth muscle [16]. It has sedative effects on the central nervous system at low dose and inhibitory effect on the central nervous system at high dose [17].

7) Caoguo (Fructus Tsaoko) The $\alpha$-pinene $\beta$-pinene of Caoguo (Fructus Tsaoko) has antitussive and expectorant effects. 1, 8-Eucalyptus has analgesic, antipyretic and antiasthmatic effects. $\beta$-pinene has strong anti-inflammatory and antifungal effects, small amount of oral has a mild diuretic effect [18] [19].

8) Banxia (Rhizoma Pinelliae preparatum) It can restrain vomiting center and stop vomiting, and has obvious effect of relieving cough [20]. It also has a wide range of anti-tumor and anti-inflammatory effects [21]. Besides, it can inhibit the secretion of gastric juice and resist gastric ulcer [21].

9) Fuling (Poria cocos) The content of $\beta$-pachyman accounted for $93 \%$ of the dry weight. It can promote cellular immunity and humoral immunity [22], protect liver injury, and has obvious diuretic effect. In addition, it can inhibit gastric ulcer, and reduce gastric secretion and free acid content [23].

10) Dahuang (Radix et Rhizoma Rhei) Rhubarb anthraquinone and aloe emodin have synergistic effect in large intestine propulsion and water secretion, which can significantly stimulate large intestine propulsion and synergistic diarrhea [24]. Emodin inhibits systemic inflammatory response [25], and has anti-acute necrotizing pancreatitis and anti-colitis effects [26]. Apart from that emoidin can inhibit the 3a ion channel of SARS-COV and HCOV-0C43 and the virus release of HCOV-0C43 [27]. Chrysophanol can inhibit the formation of biofilm and has antibacterial effect [28].

11) Huangqi (Radix Astragali) Radix Astragalus, its polysaccharide and other components can significantly improve the non-specific immune function, have the promotion or enhancement effect on humoral immunity and cellular immunity. It has an obvious stimulation effect on interferon system, has its own induced and active volatilization effect [29]. Radix Astragalus polysaccharides can prevent bleomycin-induced pulmonary fibrosis [30]. Astragaloside IV has the function of bone marrow hematopoietic, cerebral ischemia and heart protection, preventing the apoptosis of cultured rat myocardial cells induced by daunoruthromycin [31]. It also has bidirectional regulation of blood sugar, anti-fatigue, anti-hypoxia and so on [32]. 
12) Tinglizi (Lepidium apetalum Willd) It has cardiotonic action, diuretic, anti-cancer effect, relive of cough, broad-spectrum antibacterial effect, and has antibacterial effect on 20 kinds of fungi such as yeast and dozens of other strains [33].

13) Chishao (Radix Paeoniae Rubra) It can expand coronary artery, increase coronary blood flow, inhibit platelet aggregation, and prolong the time of thrombosis in vitro. Paeoniflorin has anti-tumor properties [34], showing anti-inflammatory activity in several autoimmune diseases and has neuroprotective effects [35].

\subsection{Correspondence between Signs, Symptoms and Vital Organs of COVID-19 and Pharmacological Action of TCM Decoction Pieces of "Huashi Baidu Fang"}

Pharmacology studies according to literature retrieval showed that the TCM decoction pieces in "Huashi Baidu Fang" have anti-asthmatic activity in respiratory system, antitussive pharmacological activity and expectorant effect. In digestive tract system, they have antiemetic effects, have the function of repairing gastric injury, promoting gastrointestinal functional movement. In addition, they also have liver protective activity, kidney protective effect, cardioprotective effect (Table 4).

\subsection{Important Laboratory Parameters of COVID-19 and Traditional Chinese Herbal Medicine Functional Groups with Clear Pharmacological Support}

Analyzing literatures according to the pharmacological activity, we find that the TCM decoction pieces group has clear basic experimental support for the important laboratory parameters of COVID-19. Multiple drugs in the form of functional groups work together to pharmacologically active treatment of a single disease laboratory parameter. Gan Cao (Radix Glycyrrhizae), Huoxiang (Herba Pogostemonis), Houpo (Cortex Magnoliae Officinalis), Cangzhu (Rhizoma Atractylodis), Dahuang (Radix et Rhizoma Rhei), Huangqi (Radix Astragali) and Chishao (Radix Paeoniae Rubra) have antiviral activity. Gan Cao (Radix Glycyrrhizae), Huoxiang (Herba Pogostemonis), Cangzhu (Rhizoma Atractylodis), Caoguo (Fructus Tsaoko), Tinglizi (Lepidium apetalum Willd) and Chishao (Radix Paeoniae Rubra) have extensive evidence of experimental pharmacology and clinical practice in antibacterial activity. Emodin inhibits systemic inflammation [25], and has anti-acute necrotizing pancreatitis, anti-colitis effect [26]. Honokiol has a strong inhibition of a variety of inflammatory responses [10]. And it is worth for further validation in COVID-19 (Table 5).

\section{Discussion}

Chinese medicine is based on thousands of years of history, and is an effective treatment method widely practiced by the Chinese nation in struggling against diseases. It is a scientific summary of the conditions at that time. The theory of 
Table 4. The corresponding relationship among the signs and symptoms of COVID-19, the vital organs and the pharmacological action of traditional Chinese Medicine decoction pieces of "Huashi Baidu Fang".

\begin{tabular}{|c|c|c|c|}
\hline Organs and tissues & $\begin{array}{l}\text { Clinical picture or } \\
\text { pathological changes }\end{array}$ & $\begin{array}{l}\text { Symptomatic } \\
\text { treatment }\end{array}$ & $\begin{array}{l}\text { Chinese herbal slices with corresponding } \\
\text { pharmacological evidence for important organs }\end{array}$ \\
\hline \multirow[t]{2}{*}{ Body surface } & Fever & Antipyretic & Mahuang (Herba Ephedrae), Shigao (Gypsum Fibrosum) \\
\hline & Cough & Relieving a cough & Banxia (Rhizoma Pinelliae preparatum), Tinglizi (Lepidium apetalum Willd) \\
\hline \multirow[t]{3}{*}{ Respiratory system } & $\begin{array}{l}\text { Expectoration, and } \\
\text { blood-stained sputum }\end{array}$ & Eliminating phlegm & Gan Cao (Radix Glycyrrhizae), Caoguo (Fructus Tsaoko) \\
\hline & Pant & Relieving asthma & $\begin{array}{l}\text { Mahuang (Herba Ephedrae), Xingren (Semen Armeniacae Amarum), } \\
\text { Banxia (Rhizoma Pinelliae preparatum), Tinglizi (Lepidium apetalum Willd) }\end{array}$ \\
\hline & $\begin{array}{l}\text { Gastrointestinal } \\
\text { symptoms }\end{array}$ & $\begin{array}{l}\text { Repairing gastric } \\
\text { damage }\end{array}$ & $\begin{array}{l}\text { Gan Cao (Radix Glycyrrhizae), Houpo (Cortex Magnoliae Officinalis), } \\
\text { Cangzhu (Rhizoma Atractylodis), Banxia (Rhizoma Pinelliae preparatum), } \\
\text { Fuling (Poria cocos) }\end{array}$ \\
\hline \multirow[t]{2}{*}{$\begin{array}{l}\text { Gastrointestinal } \\
\text { system }\end{array}$} & Inhibited defecation & $\begin{array}{l}\text { Promoting } \\
\text { gastrointestinal } \\
\text { functional movement }\end{array}$ & $\begin{array}{l}\text { Mahuang (Herba Ephedrae), Xingren (Semen Armeniacae Amarum), } \\
\text { Huoxiang (Herba Pogostemonis), Cangzhu (Rhizoma Atractylodi), Caoguo } \\
\text { (Fructus Tsaok), Shengdahuang (Radix et Rhizoma Rhei) }\end{array}$ \\
\hline & Emesis & Stopping vomiting & Huoxiang (Herba Pogostemoni), Banxia (Rhizoma Pinelliae preparatum) \\
\hline \multirow[t]{2}{*}{ Urinary system } & Urine is red & Diuresis & $\begin{array}{l}\text { Shengmahuang (Herba Ephedrae), Cangzhu (Rhizoma Atractylodi), Fuling } \\
\text { (Poria cocos), Shengdahuang (Radix et Rhizoma Rhei), Tinglizi (Lepidium } \\
\text { apetalum Willd) }\end{array}$ \\
\hline & Urine is red & Renal protection & Shengdahuang (Radix et Rhizoma Rhei), Shenghuangqi (Radix Astragali) \\
\hline Central system & $\begin{array}{l}\text { Abdominal } \\
\text { pain/myalgia }\end{array}$ & Analgesia & $\begin{array}{l}\text { Xingren (Semen Armeniacae Amarum), Huoxiang (Herba Pogostemoni), } \\
\text { Houpo (Cortex Magnoliae Officinalis), Caoguo (Fructus Tsaok), } \\
\text { Shengdahuang (Radix et Rhizoma Rhei) }\end{array}$ \\
\hline \multirow[t]{2}{*}{ Hepatobiliary system } & & Liver protection & $\begin{array}{l}\text { Gan Cao (Radix Glycyrrhizae), Houpo (Cortex Magnoliae Officinalis), } \\
\text { Cangzhu (Rhizoma Atractylodi), Fuling (Poria cocos), Shengdahuang } \\
\text { (Radix et Rhizoma Rhei), Chishao (Radix Paeoniae Rubra) }\end{array}$ \\
\hline & & Cholagogue & Shengdahuang (Radix et Rhizoma Rhei) \\
\hline \multirow{2}{*}{ Blood system } & & $\begin{array}{l}\text { Anti-platelet } \\
\text { aggregation }\end{array}$ & $\begin{array}{l}\text { Huoxiang (Herba Pogostemoni), Houpo (Cortex Magnoliae Officinalis), } \\
\text { Shengdahuang (Radix et Rhizoma Rhei), Shenghuangqi (Radix Astragali) }\end{array}$ \\
\hline & & Blood circulation & $\begin{array}{l}\text { Shengdahuang (Radix et Rhizoma Rhei), Shenghuangqi (Radix Astragali), } \\
\text { Chishao (Radix Paeoniae Rubra) }\end{array}$ \\
\hline \multirow{2}{*}{ The heart } & & $\begin{array}{l}\text { Cardioprotective } \\
\text { effects }\end{array}$ & $\begin{array}{l}\text { Banxia (Rhizoma Pinelliae preparatum), Shenghuangqi (Radix Astragali), } \\
\text { Tinglizi (Lepidium apetalum Willd) }\end{array}$ \\
\hline & & Adrenalin action & $\begin{array}{l}\text { Mahuang (Herba Ephedrae), Gan Cao (Radix Glycyrrhizae), Cangzhu } \\
\text { (Rhizoma Atractylodi) }\end{array}$ \\
\hline
\end{tabular}

Table 5. Important laboratory parameters for COVID-19 and pharmacologically supported traditional Chinese medicine functional group of traditional Chinese Medicine decoction pieces of "Huashi Baidu Fang".

\begin{tabular}{|c|c|c|c|}
\hline $\begin{array}{l}\text { Laboratory } \\
\text { inspection parameters }\end{array}$ & Pathology & $\begin{array}{c}\text { Pharmacology of } \\
\text { TCM decoction pieces }\end{array}$ & The name of Chinese medicine decoction piece \\
\hline $\mathrm{CRP} \uparrow$ & & & Shengmahuang (Herba Ephedrae), Xingren (Semen Armeniacae Amarum), \\
\hline $\mathrm{WBC} \uparrow$ & $\begin{array}{c}\text { Persistent } \\
\text { inflammation }\end{array}$ & Anti-inflammatory & $\begin{array}{l}\text { Gan Cao (Radix Glycyrrhizae), Houpo (Cortex Magnoliae Officinalis), Banxia } \\
\text { (Rhizoma Pinelliae preparatum), Fuling (Poria cocos), Shengdahuang (Radix et } \\
\text { Rhizoma Rhei), Shenghuangqi (Radix Astragali), Chishao (Radix Paeoniae } \\
\text { Rubra) }\end{array}$ \\
\hline $\mathrm{TNF} \uparrow$ & & Antineoplastic & $\begin{array}{l}\text { Houpo (Cortex Magnoliae Officinalis), Shengdahuang (Radix et Rhizoma } \\
\text { Rhei), Tinglizi (Lepidium apetalum Willd), Chishao (Radix Paeoniae Rubra) }\end{array}$ \\
\hline
\end{tabular}




\section{Continued}

\begin{tabular}{|c|c|c|c|}
\hline Calcitonin $\uparrow$ & $\begin{array}{l}\text { Bacterial } \\
\text { infection }\end{array}$ & Antibiosis & $\begin{array}{l}\text { Gan Cao (Radix Glycyrrhizae), Huoxiang (Herba Pogostemonis), Houpo } \\
\text { (Cortex Magnoliae Officinalis), Cangzhu (Rhizoma Atractylodis), Caoguo } \\
\text { (Fructus Tsaoko), Shengdahuang (Radix et Rhizoma Rhei), Tinglizi (Lepidium } \\
\text { apetalum Willd), Chishao (Radix Paeoniae Rubra) }\end{array}$ \\
\hline D-Dimer $\uparrow$ & $\begin{array}{l}\text { Thrombotic } \\
\text { disease }\end{array}$ & Anticoagulant & $\begin{array}{l}\text { Huoxiang (Herba Pogostemonis), Houpo (Cortex Magnoliae Officinalis), } \\
\text { Shengdahuang (Radix et Rhizoma Rhei), Shenghuangqi (Radix Astragali) }\end{array}$ \\
\hline Liver enzyme $\uparrow$ & Liver damage & Liver protection & $\begin{array}{l}\text { Gan Cao (Radix Glycyrrhizae), Houpo (Cortex Magnoliae Officinalis), } \\
\text { Cangzhu (Rhizoma Atractylodis), Fuling (Poria cocos), Shengdahuang (Radix } \\
\text { et Rhizoma Rhei), Chishao (Radix Paeoniae Rubra) }\end{array}$ \\
\hline & Virus infection & Antiviral & $\begin{array}{l}\text { Gan Cao (Radix Glycyrrhizae), Huoxiang (Herba Pogostemonis), Houpo } \\
\text { (Cortex Magnoliae Officinalis), Cangzhu (Rhizoma Atractylodis), } \\
\text { Shengdahuang (Radix et Rhizoma Rhei), Shenghuangqi (Radix Astragali), } \\
\text { Chishao (Radix Paeoniae Rubra) }\end{array}$ \\
\hline
\end{tabular}

Chinese medicine is based on the theory of Yin-yang and the five elements theory of negative and positive in "zhouyi", a traditional Chinese philosophy. In short, Yin and Yang are the two opposite sides of things. That is, the two sides of things; the theory of the five elements is actually a simple universal theory. In Chinese medicine, the etiology, symptoms, pharmacology and medicinal properties are linked in a general way based on philosophy. Traditional Chinese medicine is based on science. After nearly one hundred years of efforts, rich research results have been obtained, and people have made great progress from the chemical, pharmacological, experimental pharmacology, toxicology, pharmacodynamics and other aspects. The introduction of new concepts brings new development. But how to use the language and thinking of western medicine is a very difficult thing. At the same time, we should strive to find multiple versions of TCM classics, which are different due to different ages and inheritance reasons. Therefore, it is more authoritative and convincing to find the source of TCM prescription. We try to explain the possible value of "Huashi Baidu Fang" in the epidemic situation in an understandable way, mixed with comparative research and review, but this is a new attempt. We try to explain the unintelligible ancient medical writings of TCM in popular language; try to explain the origin of the formula by the separation of the classical prescriptions of Zhang Zhongjing's "Shanghan Lun (Treatise on Febrile Diseases)" and "Jinkui Yaolue (Synopsis of Golden Chamber)" and Wu Youxing's "Wenyi Lun (The Epidemic Febrile Disease)"; try to compare TCM symptoms with clinical manifestations and classification of Therapeutic Guidelines; try to compare the pathogenesis of the classical prescription with the diagnostic evaluation of the disease in the Therapeutic Guidelines; try to compare common pharmacological effects of a group of TCM through inquiry of pharmacology with treatment options corresponding to the guideline, although the differences of pharmacology exist between single chemical composition and mixed decoction. We attempt to expound the effectiveness and scientific nature of "Huashi Baidu Fang". We hope to bring some help for COVID-19. 
"Huashi Baidu Fang" is recommended for syndrome of lung obstruction due to epidemic toxin in "Diagnosis and Treatment Protocol for COVID-19 (Trial Version 7)" of China. The combination of the above famous prescription can be considered as the formation of "Huashi Baidu Fang". Combing the source of classical prescriptions is helpful to understand the theories of classical Chinese medicine and increase the scholars' recognition of TCM. The main clinical manifestations of syndrome of lung obstruction due to epidemic toxin are respiratory tract and gastrointestinal tract, which are compared with the acute exacerbation of COPD described in the guidelines, gastrointestinal infection described in the guidelines: acute gastroenteritis. The differentiation of clinical manifestations is helpful to the comparative analysis of western medicine and the understanding of medical workers.

"Clinical management of severe acute respiratory infection when novel coronavirus (2019-nCoV) infection is suspected-Interim guidance" made by World Health Organization (WHO) defines acute respiratory distress syndrome (ARDS). For comparison purposes, the acute exacerbations of chronic obstructive pulmonary disease (COPD) as defined in the "Therapeutic Guidelines: Respiratory (Version 5)", by Australia, were used in this study. COPD caused by COVID-19 with acute gastrointestinal inflammation is a type of disease that develops into a severe form. The literature reports that respiratory symptoms occur in $68 \%$ $75.0 \%$ of all patients [36]. 3\% - 79\% patients have gastrointestinal function affects [37], including anorexia, diarrhea, vomiting, nausea, etc. The proportion of gastrointestinal symptoms in severe patients was higher than that in non-severe patients [37], and pathology showed different degree of consolidation in lung and gastrointestinal tract [38]. Laboratory tests show the presence of a large number of inflammatory factors [39].

We should pay attention to the cytokine release syndrome (CRS) and inflammatory cytokine storm produced by increased inflammation of the gastrointestinal tract in patients with COVID-19 [39]. Increased inflammation can lead to secondary infections [40]. To treat viral gastrointestinal inflammation and respiratory inflammation, fluid rehydration and glucocorticoid include clear antivirals are used. However, the WHO document cautions against routine systemic use of glucocorticoids outside clinical trials for viral pneumonia or acute respiratory distress syndrome unless otherwise indicated. A systematic review of observational studies has shown that glucocorticoids do not improve and may harm survival in patients with acute respiratory distress syndrome. Use of glucocorticoids has both potential benefits (slightly reduced mortality) and potential negative effects (prolonged shedding of coronavirus in the respiratory tract). Modern pharmacological experiments have shown that Houpo (Cortex Magnoliae Officinalis) and Dahuang (Radix et Rhizoma Rhei) have clear and powerful anti-inflammatory factors as well as significant antibacterial and antiviral effects. They can solve the contradiction between the use of glucocorticoid and antibacterial drugs, and reduce the aggravation of disease caused by inflammatory factor outbreak and secondary infection (3.6). In this sense, TCM has natural ad- 
vantages in treating the outbreak of inflammatory factors and secondary infections.

We should also pay attention to Dayan Yin added Dahuang (Radix et Rhizoma Rhei). In "Wenyi Lun (The Epidemic Febrile Disease)", Wu Youxing discussed that during the 2 - 3 days of the initial illness, patients have fever day and night, headache and body pain, and white lichen on the tongue. He used Dayuan Yin in the morning and night to promote the downfall of evil spirits, inhibit the development of infection, stay away from the diaphragm (membrane), and expel the evil spirits from the body to achieve the purpose of treatment. If the tongue is yellow and the heart is stuffy and the abdomen is full, increase the amount of Dahuang (Radix et Rhizoma Rhei) on the basis of the Dayuan Yin in order to promote gastrointestinal drainage, remove the qi and blood stasis caused by evil qi in the gastrointestinal tract, and eliminate it from the intestinal tract. In fact, it is to inhibit gastrointestinal inflammation, remove pus blood produced by gastrointestinal inflammation, reduce intestinal endotoxin accumulation, reduce the impact on blood and viscera, and promote the recovery of gastrointestinal function. The former aims to promote the shedding of the virus in the respiratory tract, while the latter aims to promote the shedding of the virus in the intestine for elimination.

In "Shanghan Lun (Treatise on Febrile Diseases)", Zhang Zhongjing said that between 196 and 206 A.D, more than two-thirds died of 200 people in his family, $70 \%$ of them died from typhoid fever. The epidemic, which was widespread, lasted for a long time and resulted in mass death, gave birth to Zhang Zhongjing's "Shanghan Lun (Treatise on Febrile Diseases)". There were four major epidemics between 200 and 219. In 217, a major epidemic occurred in northern China, causing sickness and death in every family [41]. "Jinkui Yaolue (Synopsis of Golden Chamber)" written by Zhang Zhongjing before 219 A.D, is a summary of zhang zhongjing's decades as a senior official and medical scientist in organizing epidemic prevention and actively treating patients. Zhang Zhongjing is a Chinese medical saint. In "Wenyi Lun (The Epidemic Febrile Disease)", Wu Youxing said that in 1641, the epidemic was prevalent in Shandong, Zhejiang, Beijing, Jiangsu and Anhui, and it was more serious in May and June, and some were infected in the whole family. In south of Baoding in Hebei, Dangshan in Anhui, Suqian in Jiangsu and North of Huaiyin (the ancient route of Yellow River in Ming Dynasty), from spring to summer, there was a pandemic of plague, which killed very many people. In 1628-1644 Ming Dynasty Chongzhen years, the epidemic almost every year [42], Wu Youxing wrote "Wenyi Lun (The Epidemic Febrile Disease)" in the accumulation of long-term medical knowledge and long-term medical practice, established a new set of theories and treatment system in line with the epidemic of temperature, and founded the Dayuan Yin [43]. Chinese medicine has also gained positive experience in treating infectious diseases [44].

Although TCM for COVID-19 itself we cannot sure is restrain or kill, but from the ease of the disease caused by COVID-19, from the evidence of phar- 
macology and therapeutics, from the relieve of the suffering of the patients, from the reduce of the social health care costs, this public, cheap, consists of natural medicine, has the exact pharmacological evidence to support and long-term clinical use case plant therapy is a kind choice of the solutions. It should be expected to play a very good anti-epidemic role.

\section{Authors' Contribution}

Dandan Song participated in the discussion of conception and framework, consulted the literatures and drafted the article. Hualiang Chen, has the same contribution, proposed the conception and framework of the paper, and reviewed the article.

\section{Conflicts of Interest}

There was no conflict of interest for each named author. The authors of this article have received no financial or material support from any corporation, foundation or individual.

\section{References}

[1] Yang, X.Y., Xiao, C.F., Zhang, K.Y. and Cheng, P.J. (2015) Research Progress on Clinical Application and Pharmacological Functions of Ephedra. Chinese Archives of Traditional Chinese Medicine, 33, 2874-2877.

[2] Huang, L., Wang, Y.-N. and Wu, S.-Y. (2018) Research Progress of Pharmacological Effects of Traditional Chinese Medicines Ephedrae. China Foreign Medical Treatment, 37, 195-198.

[3] Li, L., Dai, T., Li, X., Lin, Y., Yang, M. and Zheng, M. (2016) Advances in Pharmacology of Amygdalin. Journal of Jilin Medical University, 37, 63-66.

[4] Shi, D., Liu, D., Cao, C. and Qiao, K. (2018) Research Progress on Pharmacological Action and Processing Technology of Bitter Almond. Asia-Pacific Traditional Medicine, 14, 106-109.

[5] Zhang, E.X., Yin, S.T., Zhao, S., Zhao, C., Yan, M.Z., Fan, L.H. and Hu, H.B. (2020) Protective Effects of Glycycoumarin on Liver Diseases. Phytotherapy Research, 34, 1191-1197. https://doi.org/10.1002/ptr.6598

[6] Gaur, R., Gupta, V.K., Singh, P., Pal, A., Darokar, M.P. and Bhakuni, R.S. (2016) Drug Resistance Reversal Potential of Isoliquiritigenin and Liquiritigenin Isolated from Glycyrrhiza glabra against Methicillin-Resistant Staphylococcus aureus (MRSA). Phytotherapy Research, 30, 1708-1715. https://doi.org/10.1002/ptr.5677

[7] Xu, W., Wu, Y,-Q., Ding, H.-R. and Xiu, Y.-F. (2017) Research Progress on Pharmacological Effects and Mechanism of Herba Pogostemonis. Shanghai Journal of Traditional Chinese Medicine, 51, 103-106.

[8] Shin, S. and Kang, C.-A. (2003) Antifungal Activity of the Essential Oil of Agastache rugosa Kuntze and Its Synergism with Ketoconazole. Letters in Applied Microbiology, 36, 111-115. https://doi.org/10.1046/j.1472-765X.2003.01271.x

[9] Tsai, J.-J., Chen, J.-H., Chen, C.H., Chung, J.-G. and Hsu, F.-T. (2020) Apoptosis Induction and ERK/NF- $\kappa$ B Inactivation Are Associated with Magnolol-Inhibited Tumor Progression in Hepatocellular Carcinoma in Vivo. Environmental Toxicology, 35, 167-175. https://doi.org/10.1002/tox.22853 
[10] Chen, S.-C., Chang, Y.-L., Wang, D.L. and Cheng, J.-J. (2006) Herbal Remedy Magnolol Suppresses IL-6-Induced STAT3 Activation and Gene Expression in Endothelial Cells. British Journal of Pharmacology, 148, 226-232. https://doi.org/10.1038/sj.bjp.0706647

[11] Lan, K.-H., Wang, Y.-W., Lee, W.-P., Lan, K.-L., Tseng, S.-H., Hung, L.-R., et al. (2012) Multiple Effects of Honokiol on the Life Cycle of Hepatitis C Virus. Liver International, 32, 989-997. https://doi.org/10.1111/j.1478-3231.2011.02621.x

[12] Xia, T., Zhang, J.Z., Han, L.Y., Jin, Z.X., Wang, J., Li, X., et al. (2019) Protective Effect of Magnolol on Oxaliplatin-Induced Intestinal Injury in Mice. Phytotherapy Research, 33, 1161-1172. https://doi.org/10.1002/ptr.6311

[13] Sakaue, Y., Domon, H., Oda, M., Takenaka, S., Kubo, M., Fukuyama, Y., et al. (2016) Anti-Biofilm and Bactericidal Effects of Magnolia Bark-Derived Magnolol and Honokiol on Streptococcus Mutans. Microbiology and Immunology, 60, 10-16. https://doi.org/10.1111/1348-0421.12343

[14] Hoi, C.P., Ho, Y.P., Baum, L. and Chow, A.H.L. (2010) Neuroprotective effect of Honokiol and Magnolol, Compounds from Magnolia Officinalis, on Beta-AmyloidInduced Toxicity in PC12 Cells. Phytotherapy Research, 24, 1538-1542. https://doi.org/10.1002/ptr.3178

[15] Matsui, N., Akae, H., Hirashima, N., Kido, Y., Tanabe, S., Koseki, M., et al. (2016) Magnolol Enhances Hippocampal Neurogenesis and Exerts Antidepressant-Like Effects in Olfactory Bulbectomized Mice. Phytotherapy Research, 30, 1856-1861. https://doi.org/10.1002/ptr.5695

[16] Wang, K.-T., Chen, L.-G., Wu, C.-H., Chang, C.-C. and Wang, C.-C. (2010) Gastroprotective Activity of Atractylenolide III from Atractylodes ovata on Ethanol-Induced Gastric Ulcer in Vitro and in Vivo. Journal of Pharmacy and Pharmacology, 62, 381-388. https://doi.org/10.1211/jpp.62.03.0014

[17] Deng, A.P., Li, Y., Wu, Z.T., Liu, T., et al. (2016) Advances in Studies on Chemical Compositions of Atractylodes lancea and Their Biological Activities. China Journal of Chinese Materia Medica, 41, 3904-3913.

[18] Shin, J.-S., Ryu, S., Jang, D.S., Cho, Y.-W., Chung, E.K. and Lee, K.-T. (2015) Amomum Tsao-Ko Fruit Extract Suppresses Lipopolysaccharide-Induced Inducible Nitric Oxide Synthase by Inducing Heme Oxygenase-1 in Macrophages and in Septic Mice. International Journal of Experimental Pathology, 96, 395-405. https://doi.org/10.1111/iep.12159

[19] Yang, X. (2011) Study on Extraction Technology and Pharmacological Action of Volatile Oil from Amomum tsaoko. Anhui Agricultural University, Hefei.

[20] Zhang, H., Yan, G., Lai, X. and Liu, Y. (2019) Study on Antitussive and Expectorant Effects of Pinellia ternata with Red Bud Ginger. Journal of Jiangxi University of Traditional Chinese Medicine, 31, 80-82.

[21] Zuo, J., Mou, J.G. and Hu, X.Y. (2019) Research Progress on Chemical Constituents and Modern Pharmacological Effects of Pinellia ternata. Journal of Liaoning University of Traditional Chinese Medicine, 9, 26-29.

[22] Li, X.L., He, Y.L., Zeng, P.J., Liu, Y., Zhang, M., Hao, C., Wang, H., Lv, Z.H. and Zhang, L.J. (2019) Molecular Basis for Poria cocos Mushroom Polysaccharide used as an Antitumour Drug in China. Journal of Cellular and Molecular Medicine, 23, 4-20. https://doi.org/10.1111/jcmm.13564

[23] Wu, K., Guo, C., Yang, B., Wu, X.M. and Wang, W.M. (2019) Antihepatotoxic Benefits of Poria cocos Polysaccharides on Acetaminophen-Lesioned Livers in Vivo and in Vitro. Journal of Cellular Biochemistry, 120, 7482-7488. 
https://doi.org/10.1002/jcb.28022

[24] Yagi, T., Yamauchi, K. and Kuwano, S. (1997) The Synergistic Purgative Action of Aloe-Emodin Anthrone and Rhein Anthrone in Mice: Synergism in Large Intestinal Propulsion and Water Secretion. Journal of Pharmacy and Pharmacology, 49, 22-25. https://doi.org/10.1111/j.2042-7158.1997.tb06745.x

[25] Chen, X.R., Yang, K., Jing, G.D., Yang, J. and Li, K. (2020) Meta-Analysis of Efficacy of Rhubarb Combined with Early Enteral Nutrition for the Treatment of Severe Acute Pancreatitis. Journal of Parenteral and Enteral Nutrition, 44, 1066-1078. https://doi.org/10.1002/jpen.1789

[26] Hu, J., Li, P.L. and Zhang, T. (2018) Rhubarb Combined with Trypsin Inhibitor for Severe Acute Pancreatitis: A Systematic Review and Meta-Analysis. Phytotherapy Research, 32, 1450-1458. https://doi.org/10.1002/ptr.6096

[27] Dong, X., Fu, J., Yin, X.B., Cao, S.L., Li, X.C., Lin, L.F., et al. (2016) Emodin: A Review of Its Pharmacology, Toxicity and Pharmacokinetics. Phytotherapy Research, 30, 1207-1218. https://doi.org/10.1002/ptr.5631

[28] Xie, L., Tang, H.L., Song, J.W., Long, J.Y., Zhang, L.L. and Li, X.F. (2019) Chrysophanol: A Review of Its Pharmacology, Toxicity and Pharmacokinetics. Journal of Pharmacy and Pharmacology, 71, 1475-1487. https://doi.org/10.1111/jphp.13143

[29] Fu, J., Wang, Z.H., Huang, L.F., Zheng, S.H., Wang, D.M., Chen, S.L., Zhang H.T. and Yang, S.H. (2014) Review of the Botanical Characteristics, Phytochemistry, and Pharmacology of Astragalus membranaceus (Huangqi). Phytotherapy Research, 28, 1275-1283. https://doi.org/10.1002/ptr.5188

[30] Xu, L., Li, L.-C., Zhao, P., Qi, L.-W., Li, P., Gao, J. and Fei, G.H. (2014) Total Polysaccharide of Yupingfeng Protects against Bleomycin-Induced Pulmonary Fibrosis via Inhibiting Transforming Growth Factor- $\beta 1$-Mediated Type I Collagen Abnormal Deposition in Rats. Journal of Pharmacy and Pharmacology, 66, 1786-1795. https://doi.org/10.1111/jphp.12308

[31] Luo, Z.G., Zhong, L., Han, X.F., Wang, H.R., Zhong, J.H. and Xuan, Z.H. (2009) Astragalus membranaceus Prevents Daunorubicin-Induced Apoptosis of Cultured Neonatal Cardiomyocytes: Role of Free Radical Effect of Astragalus membranaceus on Daunorubicin cardiotoxicity. Phytotherapy Research, 23, 761-767. https://doi.org/10.1002/ptr.2575

[32] Lv, L., Wu, S.-Y., Wang, G.-F., Zhang, J.-J., Pang, J.-X., Liu, Z.-Q., et al. (2010) Effect of Astragaloside IV on Hepatic Glucose-Regulating Enzymes in Diabetic Mice Induced by a High-Fat Diet and Streptozotocin. Phytotherapy Research, 24, 219-224. https://doi.org/10.1002/ptr.2915

[33] Wang, Y. and Gong, J. (2008) Chemical Constituents and Pharmacological Effects of Lepidium apetalum Willd. Journal of Changchun University of Traditional Chinese Medicine, 24, 39-40.

[34] Xiang, Y.J., Zhang, Q., Wei, S.J., Huang, C., Li, Z.S. and Gao, Y.X. (2020) Paeoniflorin: A Monoterpene Glycoside from Plants of Paeoniaceae Family with Diverse Anticancer Activities. Journal of Pharmacy and Pharmacology, 72, 483-495. https://doi.org/10.1111/jphp.13204

[35] Xu, H.Y., Cai, L., Zhang, L.L., Wang, G.J., Xie, R.L., Jiang, Y.S., Yuan, Y.Y. and Nie, H. (2018) Paeoniflorin Ameliorates Collagen-Induced Arthritis via Suppressing Nuclear Factor- $\kappa$ B Signalling Pathway in Osteoclast Differentiation. Immunology, 154, 593-603. https://doi.org/10.1111/imm.12907

[36] Zhang, J.-J., Dong, X., Cao, Y.-Y., Yuan, Y.-D., Yang, Y.-B., Yan, Y.-Q., et al. (2020) 
Clinical Characteristics of 140 Patients Infected with SARS-CoV-2 in Wuhan, China. Allergy, 75, 1730-1741. https://doi.org/10.1111/all.14238

[37] Tian, Y., Rong, L., Nian, W.D. and He, Y. (2020) Review Article: Gastrointestinal Features in COVID-19 and the Possibility of Faecal Transmission. Alimentary Pharmacology \& Therapeutics, 51, 843-851. https://doi.org/10.1111/apt.15731

[38] Bodini, G., Demarzo, M.G., Casagrande, E., De Maria, C., Kayali, S., Ziola, S., et al. (2020) Concerns Related to COVID-19 Pandemic among Patients with Inflammatory Bowel Disease and Its Influence on Patient Management. European Journal of Clinical Investigation, 50, e13233. https://doi.org/10.1111/eci.13233

[39] Shimabukuro-Vornhagen, A., Gödel, A., Subklewe, P., Schlößer, M., Schlaak, H., Kochanek, M., et al. (2018) Cytokine Release Syndrome. Journal for ImmunoTherapy of Cancer, 6, 56. https://doi.org/10.1186/s40425-018-0343-9

[40] Xu, K.J., Cai, H.L., Shen, Y.H., Ni, Q., et al. (2020) Management of COVID-19: The Zhejiang Experience. Journal of Zhejiang University (Medical Sciences), 49, 147-157.

[41] Wang, F. (2011) Epidemic Disease and Society of North China in 3rd-6th Centuries. Ph.D. Thesis, Jilin University, Jinlin.

[42] Chen, X. (2011) Plague and Society in Ming Dynasty. Xinan University, Chongqing.

[43] Wu, S. (2009) The Study on Wu Youxing's Treatise on Epidemic Febrile Diseases, Yuan Ban's Treatise on Treating Febrile Diseases and the Formation of TCM Febrile Diseases. Ph.D. Thesis, Guangzhou University of Chinese Medicine, Guangzhou.

[44] Tao, X. (2010) Study on the Origin and Treatment of Epidemic Diseases in Traditional Chinese Medicine. Nanjing University of Traditional Chinese Medicine, Nanjing. 


\section{Highlights}

- "Huashi Baidu Fang" is recommended for syndrome of lung obstruction due to epidemic toxin in "Diagnosis and Treatment Protocol for COVID-19 (Trial Version 7)". It is derived from "Shanghan Lun (Treatise on Febrile Diseases)" and "Jinkui Yaolue (Synopsis of Golden Chamber)" of Zhang Zhongjing in Han Dynasty, and "Wenyi Lun (The Epidemic Febrile Disease)" by Wu Youxing in Ming Dynasty. It is adjusted and combined on the basis of these works.

- The prescription basis and symptoms of "Huashi Baidu Fang" is basically consistent with acute exacerbation of chronic obstructive pulmonary disease (COPD) in "Therapeutic Guidelines: Respiratory (Version 5)" published by Australia, functional gastrointestinal diseases in "Therapeutic Guidelines: Gastrointestinal (Version 5)", respiratory infections: acute exacerbation of COPD in "Therapeutic Guidelines: Antibiotic (Version 15)". And the pathogeny is similar with gastrointestinal infection: acute gastroenteritis.

- It is supported by clear and extensive pharmacological research literatures to use the natural drug in "Huashi Baidu Fang" to treat acute exacerbation of COPD and functional gastrointestinal diseases in "Therapeutic Guidelines".

- We believe that in the face of the global epidemic crisis, in the case of the vaccine and effective drugs are still under research, this opened and cheap Chinese medicine consisted of the natural drug which has definite pharmacological evidence and has been used in clinical may play a positive role in the anti-epidemic. 